\title{
DEVELOPING A FRAMEWORK TO EVALUATE INDIVIDUAL LeARNing in ENgineEring DESIGN Problems - PART 1: Teaching Through Simulacra of Real-World Problems
}

\author{
Nishant Balakrishnan \\ Centre for Engineering Professional Practice and Engineering Education \\ Faculty of Engineering, University of Manitoba \\ Nishant.Balakrishnan@UManitoba.ca
}

\begin{abstract}
In the context of teaching design, engineers often have a strong preference for problem-based learning because the skills they are trying to teach are intrinsic to the solving of design problems. The proliferation of problem-based learning (PBL) in capstone and now cornerstone engineering design courses is well supported by industry and faculty and the trend has been towards seeing more PBL in engineering design courses. This paper explores the basic selection of engineering design problems and presents a fairly simple dilemma: the skills that are required to solve a problem are not necessarily the skills that are taught by the problem if the problem is truly open-ended. This paper presents the idea of using engineering problems that are carefully constructed simulacra of real-world problems with built in scaffolding to create PBL experiences for students that are educationally complete and meaningful. This paper presents examples from two courses developed at the University of Manitoba based on this approach, outcomes of and responses to the course layout, and ideas for how this model can be extended to other courses or programs.
\end{abstract}

Keywords: Problem-based learning (PBL), Engineering design, open ended design problems, experiential.

\section{INTRODUCTION}

Engineers, by nature, are problem-solvers and tend to highly value problem-based learning when trying to teach engineering students. This concept is not only a valuebased idea, but is also heavily supported by the Canadian Engineering Accreditation Board (CEAB), which requires all accredited programs to have a capstone or similar upper year design course that pulls from real world engineering problems and exposes students to the multifaceted problems they will (by nature) face as graduated engineers. These types of engineering design problems are often viewed as holistic problems that pull from a wide range of skillsets and require a fairly diverse education and practice background to solve. This idea is quite straightforward when taken at face value, but in practice there is a major complication. An engineer with a well-developed understanding of their field uses skills and knowledge from all 12 CEAB graduate attributes (GAs) to solve even simple problems, but even a holistic problem does not necessarily teach a student every attribute or explicitly require them to exercise them. An example of this can be clearly seen in [1] where engineering practitioners with experience clearly see problems from a different perspective compared to novice practitioners. In a typical capstone level course this proves to be less of a problem, since the experience and education level of students is higher. But when the same PBL strategies are tried at a lower level (typically called a cornerstone course), this tends to drastically reduce the learning outcomes students take from a course. This can be controlled to some extent by scaffolding student learning to ensure students approach and solve problems using specific skills and knowledge [2]. A drawback is that scaffolding a problem can give students and industry professionals the impression students are not being taught "real-world problems." From both of these groups, there is a push to focus on more genuine industry problems [3].

A proposed solution is to look at teaching engineering problem-solving from a different perspective: to understand the difference between creating a simulation of industry problems and creating a simulacrum. A simulated problem is an industry problem taught and approached in the same manner it would be in industry. A simulacrum problem, on the other hand, is a problem that at face value presents itself as a simulation but in actuality is a carefully crafted representation that can be modified to maximize student learning. In order to do this, both the problem and the scaffolded supporting content must be carefully developed [3] [4]. A major benefit of using simulated realworld problems is that they engage students, allowing them to frame the skills and knowledge they develop in a context that is perceived as genuine. 


\subsection{Background}

At the University of Manitoba ( $\mathrm{U}$ of $\mathrm{M}$ ), one course that heavily drew on PBL is the now discontinued MECH 2012 course, a second-year undergraduate design course that focuses on having students solve real world problems in a team and problem-based learning environment. The course has always used engineering problems which were selected based on their potential to be open-ended, challenging design problems that second year students could solve. The problems were set so that there was a mix of design, fabrication, and elements of teamwork. The problems themselves were not industry-related, and were often open-ended in many ways, but with enough constraints to force students to come up with creative solutions to the problem that emphasize skills taught in the course. Examples include line following robots, payload delivery robots, and fluid pumps. In 2015, a simple idea was explored to reconsider the direction of the course: could an industrial-focused problem be presented in such a way that the course could acquire a cornerstone feel, while still retaining the carefully scaffolded experience of the original open-ended course?

This paper outlines an example of a framework that applies to two different engineering problems through which the $\mathrm{U}$ of $\mathrm{M}$ has taught engineering design to students in both Biosystems and Mechanical Engineering. Although the framework is specific to these problems, the goal is to provide insight into how to set up a specific engineering design problem for effective PBL.

\section{METHODOLOGY}

A key aspect in developing this approach was to carefully consider one of the core tenants of problem-based learning: the problem that is selected to teach with will drive the learning process the students undertake. In a typical real-life engineering problem, there is little to no scaffolding in place and the engineer can solve the problem in whatever way best serves the problem itself. Normally this is not an issue as a practicing engineer might come up with a creative way to solve a specific problem using only a narrow sampling of their skillset (or even subcontracting the problem out), but they still maintain the broader knowledge required to solve most of the problems they're faced with.

To pose a simple example: an engineer working in an electromechanical industry is asked to design a simple enclosure $(6 \times 6 \times 6)$ with active cooling for a device emitting 200W of heat from a 2" square and determine what the cooling requirements are for the device. An engineer may choose to determine the heat-flux from the device, the convective cooling requirements, and design a device with enough cooling capacity to meet the requirements. This may be a valid solution, but what if someone poses a simple question: does there even need to be an enclosure? In a realworld problem, this is a dialogue that can easily happen: perhaps the vendor requires an enclosure, perhaps the client desires one, or perhaps it's even the company's prerogative to design enclosures so they're going to put one in regardless. But, in the real world the option to not design an enclosure and change the problem a bit to meet the solution is not only a valid approach, it's often considered an innovative and simple one. From an educational standpoint, this poses a major complication: is the problem meant to teach a student about problem-solving in general, or is it meant to test a student's skill in enclosure design? In the former case the problem is fine, but in the latter case (as would be the case in a lower year design course) this poses some major challenges.

In many real-world problems, situations like this occur all the time: in some cases, engineers will use certain skillsets to solve a problem, and in other cases they will think outside of the problem constraints and determine if a problem is solvable as constrained. In an educational setting however, this can pose some serious challenges, as each option may present a valid engineering learning outcome but both present wildly different specific outcomes. In an introductory engineering course for example, the case of redefining the problem may serve as a valid learning outcome to just that end: some engineering problems can be modified to better suit the situation at hand. In an advanced course that teaches enclosure design or thermodynamics, this would be a non-ideal outcome, as the learning objectives of the course likely do not agree with the approach taken to solve the problem.

\subsection{Simulation vs. Simulacrum}

This poses an interesting challenge to an educator, as both of these outcomes end up being vastly different approaches to solving a problem, but only one works with a focused higher year curriculum where certain learning objectives are expected from a project. The obvious solution is to constrain the problem to force students to go through certain steps to solve it. But how do you adequately constraint a problem so that it makes students approach it a certain way without losing the real-world feel that students and employers crave? One answer relies on an idea from [5], where Baudrillard describes the difference between items that are simulations of real life and items that appear to be simulations but are in fact synthetic. Beadillard talks of the simulacrum as a simulation of an item that never truly existed, but is hard to distinguish from one that does. In many ways this mirrors the ideal educational engineering problem. In most engineers' eyes, a problem is multifaceted and defined, yet at the same time open-ended. In reality, problems like this rarely exist, and simulations of real-world problems often play out like the example posed earlier in this section. Most of the perception of engineering design is actually the aggregate of a variety of design problems and experiences that challenge engineers in a multifaceted manner. One potential solution relies on trying Baudrillard's approach 
and developing a design problem that acts as a simulacrum: something that students feel and interact with as a real industry problem, but is in fact a carefully constrained problem that differs from reality.

To apply this, a simple concept was developed: problems can be devised that have an underlying evaluative framework that force students to apply and meet specific learning outcomes. Underneath these problems is a minimally constrained framework that enforces compliance with the required learning outcomes. This makes it so that the problems end up being a simulacrum, where although they are technically based on real problems, they are carefully constrained to provide specific learning outcomes, even if that means the end solutions are not complete solutions to the underlying problem.

\subsection{Course Implementation}

To apply this simulacrum approach, a framework was developed to define, select, and describe design problems in two separate second year design courses in Biosystems and Mechanical Engineering. Both courses are focused on teaching specific skills through a problem-based learning approach using semi self-directed learning [6]. Both courses cover the basics of mechanical design, use of computer-aided design, and applied design skills learned through the use of engineering tools, but the courses diverge in their broader scopes and overall learning paths. The framework was used to set up major projects that were simulacra of real-world design challenges, but with a strict scaffolding structure built into the projects themselves. The projects were created to involve all aspects of design, from definition to ideation to prototyping to testing to reflection [7].

In the Mechanical iteration of the course, students developed automated de-icing physical prototypes for High Voltage Direct Current (HVDC) transmission lines in partnership with Manitoba Hydro. This is a problem where local industry can be engaged, and students can be engaged in an issue that has a significant resonance as a "local problem." In the Biosystems course, students developed autonomous oil skimmers for algae bloom skimmers, a problem which also has significant local engagement and resonance and is relevant to the field of Biosystems engineering. While these problems are widely different, they share the same underlying constraint framework to define how students approach the problems. Though the courses were framed quite differently, they followed a comparable schedule and had similar lecture content on mechanical design and CAD software. Students designed and built prototypes which met a set of constraints crafted to specifically achieve learning outcomes attached to each course.

In the Mechanical iteration of the course, a real-world solution to mechanical de-icing may be quite varied, from a ground vehicle that is capable of mechanically removing ice from lines, to a purely electrical solution involving heating of lines. From the basis of conceptual design, this may be a good variation, but once the design passes into the detailed design phase, students' experiences would be largely shaped by the solutions they pick, and there is a large potential for the solution to have no overlap with the course content itself. In order to shape the project around this, the project was constrained to be a mechanically powered device that moves along a HVDC power line autonomously and is capable of mechanically removing ice from the line. This defines the approach students have to take, but does not define their solutions beyond that, which agrees with the perspective in [8]. The rest of the framework involves linking course learning objectives into soft constraints that the students are forced to consider as they move through their design. As an example of this, the following framework is used to build a constraint:

- Learning Objective 7: Understand and apply the concept of manufacturing tolerance to the design of mechanical parts.

- Outcome 7.1: By the end of the term, students should be able to understand how to determine the manufacturing tolerance of a specific process and be able to produce parts with a functional fit such as a clearance fit.

- Project Constraint: Provide machined (high precision) shafts components for student designs that must interface with only laser cut (moderate precision) or $3 \mathrm{D}$ printed (low precision) parts.

In this case, the constraint covers a specific learning objective in the course, under a more general learning objective, and generally forces students to apply a concept they've learnt in the context of the design problem. Since the devices the students are expected to design are purely mechanical, every single design will use shaft components in some capacity, and therefore students are always exposed to that learning outcome. In general, many constraints are lumped together to form a simpler constraint that enforces and ties a number of technical learning outcomes together. A two-page document of constraints is given to students, with some of the constraints being enforced by material or parts availability. In general, this same list of constraints can be used for almost any mechanical project to generally ensure that the course learning objectives are met, and allows for a variety of different projects to go through the general course objectives. As an example of this, both the Biosystems and Mechanical iterations of the course share the same set of general constraints for their projects (mostly), and in both cases students end up with comparable machine design skills, through their projects.

The remainder of the constraints come from the selection of the project itself, which also sets the overall difficulty of the course. For example, in the Mechanical 
iteration of the course, there is a heavier emphasis on advanced mechanical design, so the project was scaffolded to be substantially more difficult by imparting extra constraints that favour good design work. This can be done in a variety of small ways by modifying the constraints of the problem itself, without changing the overall nature of the course. For example, in the Mechanical iteration of the course, a portion of the course covers design strength and designing items so that they can be connected in a strong and rigid manner. The suspension of a device from a wire poses an inherent design conflict that students must solve: the heavier their project is the more stress the project itself must internally handle. In some years the projects are constrained so that students can suspend and drive their project on top of the wire, which minimizes this, but in other years the projects are constrained so that the project cannot reasonably interact with the top of the wire, which increases the intrinsic design requirements of the projects greatly. As an example of this, three problems that are relevant real-world problems are described below which can use the same constraint set (generally) but have vastly increasing difficulty:

1. Driving a device inside a tube to do automated inspection of DC power distribution wires within conduit.

2. Driving a device on a suspended HVDC power transmission line, with the line having simple mounts that come from the bottom of the line up to the line itself.

3. Driving a device on a suspended HVDC power transmission line, with the line having simple mounts that the line is suspended from (top mounted).

In all three cases, the same basic skillsets are tested, but students are forced to come with increasingly inventive design solutions to apply the skills they're expected to use. In all three cases, the same industry can also be engaged, and the problem can be presented in partnership with a company to establish the project's industrial and/or realworld application. In reality however, none of the three projects are set up the way that students might approach them if they were actually working for a company and tasked to solve the problem. This makes these problems a simulacrum of what you might find in industry: behind the problem itself lies a significant amount of framework and scaffolding.

\section{OUTCOMES AND RESPONSE}

From 2016 to present, the course has been offered in its current iteration twice per year in Mechanical Engineering and once per year in Biosystems Engineering. Each cohort of students has been given a different problem, with the Biosystems course focused on projects that are designed to resonate with a Biosystems specialty (oil skimmers, algae skimmers, and automated harvesters) and the Mechanical projects focusing on de-icing of various HVDC hydro lines. Each year the course has been developed further so that in the latest offering students get to see the design process through from initial concept to the presentation and testing of a full working prototype in four weeks of class time. The success rate of the prototypes tends to be on the order of $80 \%$, and every group who receives a passing mark in the course has delivered a functional prototype that has exposed them to a fairly multifaceted problem on the upper edge of what can be expected from second year students.

\subsection{Student Perspectives}

Students who were exposed to the new course (starting in 2016) were quite receptive to the content, as the course represented a major shift in covered technical content. Students were engaged in the competitive nature of the projects, enjoyed the industry engagement with the project, and appreciated the real-world feel to the problems. Feedback for the courses so far has been excellent, in many cases with the SEEQ offerings for the courses being substantially higher than the faculty/department average. Students broadly appreciated the hands-off nature of the design, where students (guided by the approach in [8]) were given significant leeway on how they approached the design as long as it was within broad constraints. Much of the feedback from the students was that they valued the course content enough to warrant a larger number of credit hours being assigned to the course, and appreciated the extended contact hours they had in the course to work on projects. As a result of this, the course (which originally started as a graphics course) was expanded and holds a matching credit hour count to the final year capstone course in the Mechanical program as the course has evolved to act as a cornerstone course in many ways.

\subsection{Industry and Staff Perspectives}

The response from industry collaborators and other staff has been excellent, with most of the comments being focused on the industrial and real-world nature of the problems being tackled. Since most of the industry and staff who are exposed to the course are engineers, the idea that the course touches on most aspects of engineering seems to resonate with them. To date no negative feedback has been provided about the course.

Academic staff in upper year courses have appreciated that students have been exposed to a full featured mechanical design. In the previous iteration of the course, students were able to work in premanufactured parts or could purposefully design to avoid mechanical parts, meaning all students were not exposed to mechanical design. Due to the "constraint but open-ended" approach in the redeveloped course, all students are consistently exposed to the same learning outcomes. 


\section{FUTURE WORK AND SUGGESTIONS}

As the course develops in future, the plan is to slowly expand the scope in both courses to allow students more options and more experiential learning opportunities. Examples of this include the potential to let students use alternative software and manufacturing processes that they currently are constrained away from using. There is also the potential to allow the use of machined parts, more types of digital fabrication equipment, and more exposure to the modern metrology equipment that has been made available to students.

\subsection{Suggestions and Plans for Expansion}

Engineering students outside of Mechanical and Biosystems have requested that their departments offer a similar second year course. They want to further develop their engineering and technical skills, while also taking on an opportunity to practice their design skills. The course was tailored around mechanical design, as the natural way to learn to use computer-aided design software is to design parts and devices. Not every engineer strictly needs this skill, so to apply the same concept to other disciplines may require a fairly different approach. Based on the same principles presented here however, and a different set of learning objectives, it would be possible to adapt this framework to other disciples such as electrical engineering. As a simple example of this, a similar but more ad-hoc framework is used in a pair of Mechanical upper year technical electives at the University of Manitoba that explore topics in computer-integrated manufacturing and automation, with the constraints shifted towards teaching control system design and layout. Work is currently being done to apply this framework to that course to explore how the framework can be adapted to bring constrained simulacra of real-world issues into other courses.

\section{CONCLUSIONS}

One fairly basic suggestion that comes out of this work is to think about the learning paths in courses that use PBL and to think about the application of an underlying evaluative framework to constrain and scaffold the process so that students get consistent exposure to learning outcomes. In many instances, it seems engineers are keen to engage in teaching with PBL, but unless the problems are carefully selected, the learning outcomes students are exposed to can vary greatly. The core idea of picking problems to be a simulation of what industry problems are like can often conflict with the need to teach design in a multifaceted manner. By using a simulacrum approach, problems are framed in a way that can feel quite genuine. The multifaceted nature of a full design-build is captured without the pedagogical challenges associated with using a truly real-world problem in an accredited educational program.

\section{Acknowledgements}

I would like to acknowledge the contributions of D. Kuhn of the Mechanical Engineering Department at the University of Manitoba and both D. Ruth and M. Friesen (and both of their respective NSERC Chairs in Design Engineering) of the Centre for Engineering Professional Practice for their support of both the Mechanical and Biosystems courses. I'd also like to thank J Seniuk-Cicek and R. Balakrishnan for their guidance on the paper.

\section{References}

[1] Cynthia J. Atman, et al, "Engineering design processes: a comparison of students and expert practitioners," Journal of Engineering Education, vol. 96, no. 4, pp. 359-379, 2007.

[2] Tony Greening, "Scaffolding for success in problembased learning," Medical Education Online, vol. 3, no. 1, art. 4297, 1998.

[3] Aditya Johri and Barbara M. Olds, Cambridge Handbook of Engineering Education Research. Cambridge, UK: Cambridge University Press, 2014, 792 pp. \{ISBN: 978-1107014107\}

[4] Brigid J.S. Barron, et al. "Doing with understanding: lessons from research on problem- and project-based learning," Journal of the Learning Sciences, vol. 7, no. 3-4, pp. 271-311, 1998.

[5] Jean Baudrillard, Simulacra and Simulation. Ann Arbor, MI: University of Michigan Press, 2005, 176 pp. \{ISBN: 978-0472065219\}

[6] Nicholas M. Massa, "Problem-based learning (pbl): a real-world antidote to the standards and testing regime," New England Journal of Higher Education, vol. 22, no. 4, pp. 19-20, 2008.

[7] Clive L. Dym, et al, "Engineering design thinking, teaching, and learning," Journal of Engineering Education, vol. 94, no. 1, pp. 103-120, 2005.

[8] Alice Y. Kolb and David A. Kolb. "Learning styles and learning spaces: enhancing experiential learning in higher education," Academy of Management Learning \& Education, vol. 4, no. 2, pp.193-212, 2005. 